\title{
¿CÓMO CONTABILIZAR EL IMPACTO AMBIENTAL DE LAS EMPRESAS? EL CASO DE LAS EMISIONES DE GASES DE EFECTO INVERNADERO
}

\author{
Silvia Ayuso Siart (ESCI, Universidad Pompeu Fabra) ${ }^{1}$
}

\section{Resumen:}

El objetivo del presente trabajo es analizar cómo valorar el impacto ambiental de las empresas desde una perspectiva de contabilidad social. El estudio teórico contribuye a la literatura sobre contabilidad social y medioambiental y aporta una orientación práctica sobre cómo contabilizar el impacto ambiental de las organizaciones. Tras reflexionar si el medio ambiente puede ser considerado un grupo de interés y cómo se plantea el análisis del valor ambiental creado o destruido, el trabajo se limita al caso de las emisiones de gases de efecto invernadero, principal fuente del cambio climático, y por lo tanto uno de los grandes desafíos ambientales a los que se enfrenta nuestra sociedad. En concreto, se analiza la cuantificación y valoración de las emisiones de gases de efecto invernadero mediante diferentes modelos disponibles para asignar un valor monetario, desde precios de mercado a costes de mitigación o costes sociales. El trabajo concluye que la valoración monetaria del impacto ambiental puede integrarse en la contabilidad social, siempre y cuando se considere toda la cadena de valor y los costes (o beneficios) para la sociedad. La contabilización del valor ambiental de las empresas puede ayudar a transformar la contabilidad financiera convencional y mejorar la toma de decisiones interna de las empresas de acuerdo con principios de ética y sostenibilidad.

Palabras clave: contabilidad social, valor social, stakeholders, medio ambiente, cambio climático.

Códigos JEL: M14, M41, Q54, Q56.

\begin{abstract}
:
The aim of this paper is to analyse how to assess the environmental impact of companies from a social accounting perspective. The theoretical study contributes to the literature on social and environmental accounting and provides practical guidance on how to account for the environmental impact of organizations. After reflecting on whether the environment can be considered a stakeholder and how to approach the analysis of the environmental value created or destroyed, the study is limited to the case of greenhouse gas emissions, main source of climate change, and therefore one of the great environmental challenges faced by our society. Specifically, it analyses the quantification and valuation of greenhouse gas emissions through different models available to assign a monetary value, from market prices to mitigation costs or social costs. The study concludes that monetary valuation of the environmental impact can be integrated into social accounting, as long as the entire value chain and societal costs (or benefits) are considered. Accounting for the environmental value of companies can help transform conventional economic-financial accounting and improve the internal decision making of companies in accordance with principles of ethics and sustainability.
\end{abstract}

Keywords: social accounting, social value, stakeholders, environment, climate change.

JEL Codes: M14, M41, Q54, Q56.

\footnotetext{
${ }^{1}$ silvia.ayuso@esci.upf.edu, Cátedra Mango de RSC, ESCI, Universidad Pompeu Fabra. Recibido: 17 de marzo de 2020. Aceptado: 10 de julio de 2020.
} 


\section{INTRODUCCIÓN}

Las interacciones de las empresas con el medio ambiente no se reflejan actualmente en la contabilidad financiera. Las empresas son responsables en gran medida del consumo excesivo de los recursos naturales y la degradación del entorno natural, sin que esto se refleje en su cuenta de resultados o su balance general. La falta de valoración de este “capital natural” ha sido una de las principales razones que ha llevado a sobrepasar los límites ecológicos y a operar más allá de la capacidad de carga de nuestro planeta (WWF, 2018). La atribución de un valor monetario al impacto medioambiental o ambiental permite a las empresas visibilizar su interacción con la naturaleza y facilita su consideración en la toma de decisiones. En última instancia, ayuda a las empresas a poner en práctica el compromiso ético con el medio ambiente, las poblaciones afectadas por la degradación del mismo y las futuras generaciones que lucharán contra el cambio climático y la escasez de recursos.

Sin embargo, no existen directrices reconocidas sobre cómo atribuir un valor monetario a los diversos impactos ambientales producidos por las empresas. Mientras a nivel mundial más de 3.500 grandes empresas divulgan información relativa a su comportamiento ambiental, de acuerdo con iniciativas como la Global Reporting Initiative (GRI), el International Integrated Reporting Council (IIRC) y los Objetivos de Desarrollo Sostenible de Naciones Unidas (KPMG, 2017), únicamente unas pocas empresas pioneras explicitan, cuantifican o monetizan los efectos causados sobre el medio ambiente. Un reciente estudio identifica solamente 42 casos de compañías a nivel mundial que han intentado valorar algunos de sus impactos ambientales en términos monetarios y a través de métodos diferentes (Pritchard y Van der Horst, 2018).

El objetivo del presente trabajo es analizar cómo valorar el impacto ambiental de las empresas desde una perspectiva de contabilidad crítica. Siguiendo las propuestas normativas de la contabilidad social y ambiental, argumentamos que los sistemas contables deben proporcionar información sobre el valor generado por una organización para todos sus grupos de interés. En este sentido, el trabajo explora si el medio ambiente puede ser considerado un grupo de interés y cómo se plantea el análisis del valor ambiental creado o destruido. Para profundizar en el asunto, nos centramos en la cuantificación y valoración monetaria de las emisiones de gases de efecto invernadero, principal fuente del cambio climático, y por lo tanto uno de los grandes desafíos ambientales a los que se enfrenta nuestra sociedad.

El trabajo realiza tanto aportaciones teóricas como prácticas. Por una parte, el trabajo contribuye a la literatura sobre contabilidad social y medioambiental, un área de investigación relativamente reciente (Larrinaga et al., 2019). En concreto, responde al reto de una investigación interdisciplinar, incorporando los conocimientos de las ciencias naturales y la visión sistémica del medio ambiente en los sistemas contables (Bebbington y Larrinaga, 2014; Williams et al., 2017). Por otra parte, el trabajo pretende participar activamente en el proceso de diseño de sistemas de contabilidad social y medioambiental (Gray, 2002, Parker, 2005), y aporta una orientación práctica sobre cómo contabilizar el impacto ambiental de las empresas y otras organizaciones. A la espera de normas y estándares que definan la manera de medir y comunicar el valor ambiental creado o destruido, se presentan algunos principios elementales para asegurar la rigurosidad de estas valoraciones.

Al tratarse de un estudio teórico, la metodología de la investigación ha consistido en la revisión de la literatura disponible sobre el tema. En concreto, se consultaron varias bases de datos de publicaciones académicas y se realizó una búsqueda exhaustiva en Internet. Por razones prácticas, el trabajo se ha limitado a analizar en profundidad la cuantificación y valoración monetaria de un determinado aspecto ambiental: las emisiones de gases de efecto invernadero. 
El trabajo sigue la siguiente estructura: a continuación se introduce la contabilidad social y ambiental. Posteriormente se reflexiona si el medio ambiente puede considerarse un stakeholder y cómo se lleva a cabo el análisis del valor ambiental. En el siguiente apartado se analiza la cuantificación del valor ambiental relacionado con las emisiones de gases de efecto invernadero, presentando los tres enfoques principales para asignarles un valor monetario. Finalmente, se cierra el trabajo con las conclusiones que se derivan del estudio.

\section{CONTABILIDAD SOCIAL Y AMBIENTAL}

La contabilidad es una disciplina utilizada tradicionalmente en el campo económico, con el objetivo de recopilar, analizar y sintetizar información de carácter financiero para ser presentada a diferentes usuarios de interés. Las primeras propuestas de complementar la información financiera con cuestiones sociales y ambientales surgieron en los años setenta y ochenta del siglo pasado (Larrinaga et al., 2019). La investigación desarrollada a partir de ese momento en el campo de la contabilidad social y ambiental se ha basado en una crítica a las limitaciones de la contabilidad financiera, en particular al rango limitado de elementos que considera, a su exclusión de elementos que no tienen un valor monetario y a su enfoque dirigido a accionistas y otros proveedores de capital (Gray et al., 1996). Aunque existen diferentes definiciones de la contabilidad social y ambiental, todas enfatizan generalmente la ampliación de los elementos bajo consideración (externalidades sociales y ambientales de las organizaciones) e incluyen una gama más amplia de partes interesadas como empleados, usuarios o clientes, comunidad, administraciones públicas, etc. (Richmond et al., 2003).

Una gran parte de los trabajos en el área de la contabilidad social y ambiental han analizado las prácticas de divulgación de información de carácter social y ambiental por parte de empresas y otras organizaciones (Larrinaga et al., 2019). En particular, con respecto a las cuestiones ambientales, ha habido una serie de iniciativas de organismos normalizadores para establecer la obligatoriedad de incorporar determinada información en los informes financieros (Fernández et al., 2018; Larrinaga et al., 2002). En este sentido, numerosos estudios han analizado los efectos de introducir nuevas regulaciones con respecto a la divulgación de la información contable relativa al medio ambiente (p.ej. Barbu et al., 2014; Bebbington et al., 2012; Llena et al., 2007). En líneas generales, estos trabajos concluyen que las regulaciones han aumentado la cantidad de la información ambiental divulgada por las compañías, pero que sigue siendo limitada la calidad de esta información y su utilidad para la toma de decisiones. Por otra parte, también han sido objeto de análisis las prácticas de divulgación de información ambiental y social de acuerdo con iniciativas internacionales de carácter voluntario, como la Global Reporting Initiative (GRI), el International Integrated Reporting Council (IIRC) y los Objetivos de Desarrollo Sostenible de Naciones Unidas (p.ej., Hummel, 2019; Michelon et al., 2015; Pistoni et al., 2018). Estas investigaciones revelan que la calidad general de la información publicada por las empresas aún es baja y responde más a prácticas simbólicas que sustantivas. Además, la mayoría de estas prácticas de revelación de información ambiental y social están concebidas como un complemento a los estados financieros, en un lenguaje diferente al financiero y sin realizar una valoración (monetaria) de los resultados ambientales y sociales (Retolaza y San-Jose, 2018).

En este escenario, han surgido una serie de propuestas para ir más allá del reporting no financiero y contabilizar los impactos causados por las actividades de las empresas y organizaciones mediante una valoración monetaria de estos impactos (Nicholls, 2020). Una de ellas es la metodología desarrollada por Retolaza et al. (2016) del análisis del Valor Social Integrado (VSI). La metodología se fundamenta en la perspectiva de la teoría de los grupos de interés o stakeholders (Freeman, 1984; Freeman et al., 2010). Desde esta perspectiva, se plantea adoptar un concepto más amplio del valor creado por una organización - por un lado, tomando 
en consideración el valor distribuido a todos los stakeholders de la organización y no sólo a sus propietarios o accionistas (shareholders), y por otro lado, incorporando los efectos no económicos sobre los diversos stakeholders - (Retolaza y San Jose, 2011). Esto da lugar a una visión más completa del valor generado, a la que se le puede denominar triple cuenta de resultados (triple bottom line) (Elkington, 1997), blended value (Emerson et al., 2003), valor compartido (shared value) (Porter y Kramer, 2011) o Valor Social Integrado.

El sistema de contabilidad social propuesto se basa en el llamado modelo poliédrico, que permite objetivar y visualizar el valor que crea una organización para todos sus grupos de interés (Retolaza et al., 2016). El VSI se compone del valor social generado a través de la actividad económica y el valor social que la organización genera de manera específica para sus diversos grupos de interés mediante relaciones ajenas a las transacciones de mercado. Para determinar el valor social específico o valor de no mercado se combina un análisis cualitativo y cuantitativo (Ayuso, 2018). El análisis cualitativo busca identificar el valor que la organización genera para sus grupos de interés más allá de la actividad económica, basándose en un proceso de consulta con representantes de los stakeholders sobre su percepción del valor generado. El análisis cuantitativo pretende cuantificar el valor percibido a través del desarrollo de indicadores vinculados a proxies (aproximaciones) financieras, que permitan monetizar el valor generado.

La contabilidad social del VSI se ha aplicado empíricamente a más de 50 organizaciones españolas de diferentes tamaños y sectores, tanto lucrativas como sin ánimo de lucro (Retolaza y San-Jose, 2018). La experiencia muestra que se trata de una metodología que proporciona información útil para las organizaciones que la aplican, en cuanto que ofrece una valoración del impacto generado sobre los diferentes grupos de interés (Lazkano y Beraza, 2019; Retolaza y San-Jose, 2018). No obstante, el proceso seguido de interacción con representantes de los stakeholders para averiguar su percepción del valor generado deja fuera de consideración el impacto causado por la organización sobre el medio ambiente. El presente trabajo se centra en cómo integrar la valoración monetaria de los impactos ambientales en el modelo de la contabilidad social del VSI.

\section{EL MEDIO AMBIENTE COMO STAKEHOLDER}

La metodología del VSI se basa en un planteamiento de las organizaciones bajo el prisma de la teoría de los grupos de interés, que considera la importancia de todos los stakeholders en la generación y distribución de valor (Phillips et al., 2003). En la literatura académica se ha debatido si, junto a empleados, clientes, proveedores, competidores, reguladores, etc., el medio ambiente también puede ser considerado un stakeholder. Así, Starik (1995) argumenta que las organizaciones están moralmente obligadas a respetar los recursos proporcionados y las limitaciones que presenta el medio ambiente y, en el mismo sentido, Bazin y Ballet (2004) afirman que el entorno natural tiene "condición ética" porque los humanos tienen un interés en preservar la vida que depende del medio natural. Una de las complicaciones planteadas al considerar el entorno natural como un stakeholder es que no tiene una conciencia y no puede tener necesidades como las entienden los humanos (Orts y Strudler, 2002; Phillips y Reichart, 2000). Sin embargo, Stone (1974) aboga por los derechos legales de los objetos naturales señalando que las organizaciones también son entidades no humanas, aunque se les hayan otorgado muchos derechos humanos. Más habitual es el argumento de señalar la dependencia que tienen las organizaciones del medio ambiente, dada la importancia de los recursos y los insumos económicos que proporciona (Driscoll y Starik, 2004; Starik, 1995). Un aspecto asociado con la dependencia de las organizaciones del entorno natural es el argumento esgrimido de que el medio ambiente cumple con el criterio de la definición de Freeman de los stakeholders como aquellos "que pueden afectar o son afectados por la consecución de los 
objetivos empresariales” (Starik, 1995). Finalmente, hay autores que mantienen que la inclusión del medio ambiente bajo la categoría de stakeholder amenaza el sentido mismo de la teoría de los grupos de interés (Orts y Strudler, 2002; Phillips y Reichart, 2000), mientras que otros afirman que un alcance más amplio convierte la teoría en más completa (Driscoll y Starik, 2004).

Más allá del argumento ético a favor del medio ambiente como un stakeholder al que las organizaciones deben prestar atención, Haigh y Griffiths (2009) muestran su relevancia desde una perspectiva estratégica. Basándose en el ejemplo del cambio climático, argumentan de manera convincente que el medio ambiente cumple con los atributos propuestos por Mitchell et al. (1997) y Driscoll y Starik (2004) de poder, legitimidad, urgencia y proximidad, y de esta manera se convierte en un stakeholder principal. El hecho que el medio ambiente no tenga una voz propia no significa que no se manifieste: el cambio climático, el deterioro de la capa de ozono y la reducción de recursos naturales son ejemplos de cómo el planeta expresa el impacto negativo producido por la actividad humana, y que a su vez afecta a las organizaciones, limitando sus actividades y generando incertidumbre acerca del futuro de éstas.

Sin embargo, sin voz no es posible que el medio ambiente nos comunique sus intereses y su percepción del valor creado o destruido por las organizaciones. En su lugar, deberemos recurrir al conocimiento científico para determinar los efectos causados por la actividad humana sobre el entorno natural. En los últimos años se ha incrementado la investigación con respecto a la comprensión científica de las vías de impacto sobre el medio ambiente y las personas. Un buen ejemplo es la ciencia climática y la propuesta a las empresas de definir objetivos basados en la ciencia (Science Based Targets), es decir, metas de reducción de emisiones de gases de efecto invernadero que contribuyan a prevenir las peores consecuencias del cambio climático (Faria y Labutong, 2020).

\section{ANÁLISIS DEL VALOR AMBIENTAL}

Existen numerosas herramientas de análisis ambiental utilizadas por organizaciones para inventariar y comunicar sus impactos sobre el entorno. Una iniciativa reciente es el Protocolo de Capital Natural (Natural Capital Protocol) desarrollado por la Natural Capital Coalition con el objetivo de crear un marco estandarizado global para que las empresas identifiquen, midan y valoren sus impactos y dependencias del capital natural e incluyan esta información en la toma de decisiones (NCC, 2016). ${ }^{2}$ El Protocolo también proporciona orientación sobre la valoración monetaria de los impactos sobre el medio ambiente, que ha sido utilizada en análisis de costebeneficio o en combinación con enfoques como el análisis del ciclo de vida (ACV) o las tablas input/output extendidas ambientalmente (Extended Input-Output tables) (Danish Environmental Protection Agency, 2014). En el año 2011, la compañía alemana de artículos deportivos PUMA presentó la primera cuenta de pérdidas y ganancias ambientales (EP\&L, por las siglas en inglés de Environmental Profit and Loss Account), con la finalidad de contabilizar "el importe a pagar si el medio ambiente facturara por proporcionar agua y aire limpios, restaurar los suelos y la atmósfera y descomponer los residuos” (Van Kampen, 2018, p. 2). ${ }^{3}$ Desde entonces, otras empresas han seguido el ejemplo y han elaborado EP\&L (Novo Nordisk, Kering - anterior compañía matriz de PUMA -, Philips, etc.). Los elementos básicos de una EP\&L son la evaluación ambiental y la valoración del impacto de las actividades de una

\footnotetext{
${ }^{2}$ Cabe señalar que también existen varias herramientas de medición del impacto social, algunas de las cuales incluyen aspectos ambientales en su concepto de impacto social (Ayuso, 2018). Sin embargo, se trata de enfoques que tratan el valor ambiental desde el valor percibido por las personas y no desde la visión de las ciencias naturales. ${ }^{3}$ Existen experiencias anteriores que tratan de realizar una cuenta de pérdidas y ganancias para el medio ambiente, como por ejemplo, la de la compañía sueca Fortia (Gröjer y Stark, 1977).
} 
organización y su cadena de valor (véase Figura 1). En primer lugar, se cuantifican las emisiones ambientales o el uso de recursos por parte de la entidad en unidades biofísicas (kilogramos, litros, hectáreas, etc.). A continuación, se estiman las consecuencias de estas emisiones y el uso de recursos en el entorno natural, y se valoran los impactos que se derivan para las personas en términos monetarios.

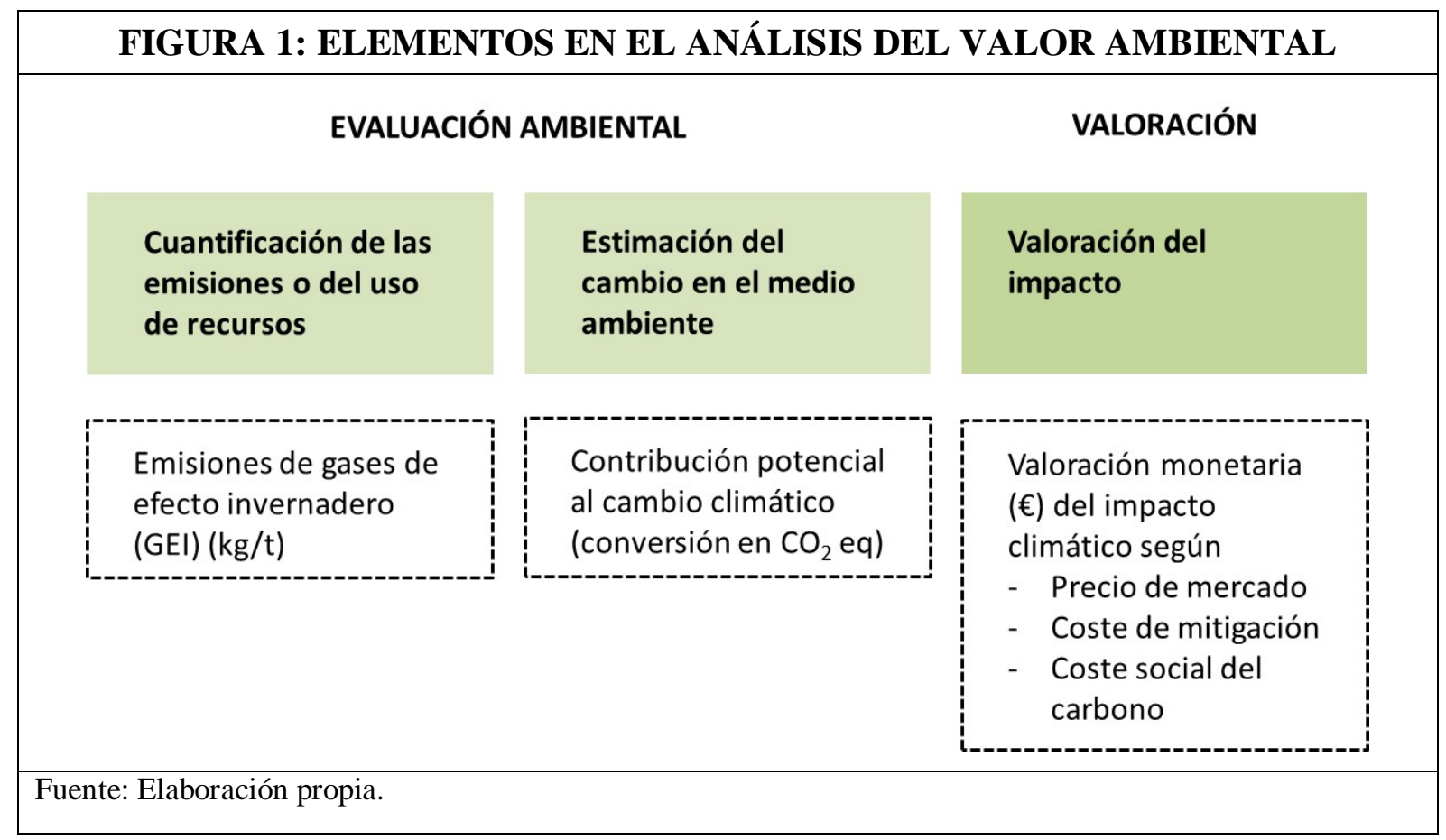

Los pasos de la EP\&L son paralelos a la fase de cuantificación y monetización del valor social percibido por los stakeholders (Retolaza et al., 2016). En primer lugar, se identifican indicadores relacionados con las categorías de valor percibido por los stakeholders, las llamadas variables de valor, que sean capaces de medir los productos y servicios tangibles resultantes de las actividades realizadas por la organización (outputs). En segundo lugar, se identifican posibles aproximaciones al valor o proxies que permitan estimar el valor monetario de los outputs generados por la organización, utilizando la lógica de sustitución (valor monetario de productos o servicios sustitutivos) o la lógica de mitigación/prevención (valor monetario de costes evitados). Finalmente, se determinan las fórmulas de cálculo que relacionan los outputs con las proxies a fin de cuantificar el valor monetario de cada variable de valor para el período de referencia.

Al igual que en el análisis del VSI, la EP\&L contabiliza el valor monetario "bruto" generado por las actividades realizadas por una organización durante un período de tiempo determinado, tomando como referencia un escenario hipotético en el que se supone que la organización no existiera y ninguna otra organización tomara su lugar (Kering, 2015). Sin embargo, a diferencia de que una parte importante del valor social generado por las organizaciones resulta beneficioso para sus grupos de interés, el valor ambiental atribuible a las actividades de las organizaciones suele ser perjudicial para el medio ambiente. Como resultado, en la mayoría de las organizaciones el valor social generado será positivo (valor creado) y el valor ambiental generado será negativo (valor destruido). Este es el caso, por ejemplo, de los resultados de la primera EP\&L de PUMA. En concreto, del valor negativo de 145 millones de euros, sólo 8 millones de euros (6\%) provienen de las operaciones propias de PUMA como oficinas, almacenes, tiendas y logística, y los restantes 137 millones de euros (94\%) están 
asociados con la cadena de suministro, sobre todo con la producción de materias primas en el nivel 4 de proveedores (PUMA, 2011).

No obstante, aunque el valor ambiental "bruto" de la organización represente una pérdida en términos contables, puede ser compensado por una ganancia ambiental inducida por la actividad. Esto es el caso del resultado de la EP\&L de Vodafone Países Bajos (Vodafone Netherlands, 2015). La compañía operadora de telefonía móvil calculó un valor ambiental negativo de 21,6 millones de euros, tomando en consideración el ciclo de vida de cuatro ámbitos de impacto: los edificios en los que opera, la red que utiliza para sus servicios, la atención al cliente realizada por los empleados y los impactos de los productos y servicios ofrecidos. Por otra parte, contabilizó un valor ambiental positivo de 37,4 millones de euros, que se deriva de reducir las emisiones de carbono de los clientes como consecuencia de los productos y servicios relacionados con la desmaterialización (telepresencia móvil, oficina virtual y notificaciones de entrega móvil para comercio electrónico) y las aplicaciones inteligentes (conectividad machine2-machine). La compañía reconoce que se trata de un balance teórico: "Si bien el impacto negativo se crea realmente, el impacto positivo se basa en evitar el impacto ambiental negativo por parte de los clientes que utilizan los productos y servicios de Vodafone. Por lo tanto, esto no es una ganancia real, sino más bien 'qué pasaría si nuestros clientes no usaran estos productos y servicios”’ (Vodafone Netherlands, 2015, p. 27).

En cualquier caso, queda patente la importancia de que la evaluación efectuada considere toda la cadena de valor. Una razón es que, en función del sector de actividad, los problemas ambientales más relevantes pueden darse en etapas anteriores o posteriores a las operaciones realizadas directamente por la organización bajo análisis - aguas arriba (upstream) o aguas abajo (downstream). Por ejemplo, la industria extractiva experimenta el mayor impacto ambiental en cuanto a la ocupación de suelo y efecto sobre la biodiversidad en sus operaciones directas y aguas arriba, mientras que las emisiones de carbono son mayores en las etapas aguas abajo. Otra razón para aplicar una evaluación integrada desde la perspectiva de toda la cadena de valor (o ciclo de vida) es para evitar lo que se conoce en las ciencias ambientales como la transferencia de cargas ambientales entre etapas de ciclo de vida, áreas geográficas o vectores ambientales (Fullana y Puig, 1997).

En el siguiente apartado analizaremos el valor ambiental relacionado con la emisión de gases de efecto invernadero, el aspecto ambiental más comúnmente contabilizado de forma monetaria por las empresas y muchas veces responsable de la mayor "pérdida" ambiental en términos contables (Pritchard y Van der Horst, 2018). Asimismo, se trata de uno de los impactos ambientales que presenta las metodologías de evaluación más sólidas, considerando la extensión de la literatura académica, el grado de consenso en esta literatura y su aplicabilidad a la medición o valoración de los impactos (PwC, 2015). Finalmente, se trata de un impacto ambiental que debido a su naturaleza global puede valorarse de manera homogénea a nivel mundial, mientras que otros impactos ambientales deben valorarse tomando en consideración los contextos nacionales o locales específicos, como, por ejemplo, en el caso del consumo de agua.

\section{VALORACIÓN DEL IMPACTO DE LAS EMISIONES DE GASES DE EFECTO INVERNADERO}

\subsection{Evaluación ambiental: inventario de las emisiones de gases de efecto invernadero}

En los últimos años ha crecido la preocupación por el cambio climático en nuestra sociedad. Como consecuencia, se han alcanzado diferentes acuerdos entre los Estados para reducir las emisiones de gases de efecto invernadero (GEI), como el Protocolo de Kioto o el 
más reciente Acuerdo de París de la Convención Marco de las Naciones Unidas sobre el Cambio Climático. Los principales gases que contribuyen al efecto invernadero son dióxido de carbono $\left(\mathrm{CO}_{2}\right)$, metano $\left(\mathrm{CH}_{4}\right)$, óxido nitroso $\left(\mathrm{N}_{2} \mathrm{O}\right)$, hidrofluorocarbonos (HFCs), perfluorocarbonos (PFCs) y hexafluoruro de azufre $\left(\mathrm{SF}_{6}\right.$ ) (IPCC, 2007). Calcular las emisiones de GEI que son provocadas tanto directa como indirectamente por las actividades de una organización equivale a calcular la llamada "huella de carbono". La huella de carbono se mide en kilogramos o toneladas de $\mathrm{CO}_{2}$ equivalente $\left(\mathrm{CO}_{2} \mathrm{eq}\right.$ o $\left.\mathrm{CO}_{2} \mathrm{e}\right)$, considerando la totalidad de las emisiones de GEI y “convirtiéndolas” en equivalentes a toneladas de $\mathrm{CO}_{2}$ en función de su potencial de calentamiento global relativo (obtenido de la comparación del efecto de las moléculas de cada uno de los gases con el efecto de la molécula de $\mathrm{CO}_{2}$ ) (véase Figura 1).

En los últimos años se han desarrollado diversos estándares que permiten realizar el cálculo de la huella de carbono tanto a nivel organizativo como de producto o servicio. La referencia más ampliamente utilizada para el cálculo de la huella de carbono de una organización es el Greenhouse Gas Protocol (GHG Protocol) (WRI y WBCSD, 2004). El GHG Protocol clasifica las emisiones de GEI en tres tipos de alcance: alcance 1 (emisiones directas de GEI de fuentes que son propiedad o están controladas por la organización), alcance 2 (emisiones indirectas de GEI asociadas a la generación de electricidad adquirida y consumida por la organización) y alcance 3 (otras emisiones indirectas ocasionadas en la cadena de valor de la organización, tanto aguas arriba como aguas abajo) (WRI y WBCSD, 2004).

Para valorar los impactos ambientales de las emisiones de GEI, debemos entender cómo estas emisiones atmosféricas afectan al entorno natural y a las personas. Está bien establecido el vínculo causal entre las emisiones antropogénicas de GEI y los cambios en el clima global. Los efectos resultantes en el medio ambiente incluyen, por ejemplo, el aumento del nivel del mar y una mayor incidencia de eventos climáticos extremos, y las consecuencias para la sociedad van desde impactos en la salud humana a distorsiones en la economía (IPCC, 2014). En la literatura sobre la valoración monetaria de los impactos climáticos se diferencian tres enfoques principales (Dong et al., 2019; Isacs et al., 2016; Mandell, 2011): el enfoque del precio de mercado, el enfoque del coste de mitigación y el enfoque del coste social del carbono (véase Figura 1). A continuación, se describen estos tres enfoques para asignar un valor monetario a los impactos causados por las emisiones de GEI.

\subsection{Valoración monetaria según el precio de mercado}

Según el enfoque del precio de mercado, la valoración de las emisiones de GEI puede derivarse de los precios de mercado inducidos por los gobiernos a través de un régimen de comercio de derechos de emisión (ETS, por las siglas en inglés de Emissions Trading Scheme) o los impuestos sobre el carbono. Un ETS, a veces denominado también sistema de limitación y comercio, fija un límite máximo para el nivel total de emisiones de GEI, y permite que las industrias con bajas emisiones vendan los derechos de emisión que les sobran a industrias con mayores emisiones (Comisión Europea, 2020). Mediante la creación de una oferta y una demanda de derechos de emisión, un ETS establece un precio de mercado para las emisiones de GEI. En cambio, un impuesto sobre el carbono establece una tasa impositiva sobre las emisiones de GEI, o, más comúnmente, sobre el contenido de carbono de los combustibles fósiles. La diferencia con un ETS es que se define directamente el precio del carbono.

En la actualidad los impuestos al carbono se aplican en diversos países y regiones, con unos rangos que oscilan desde menos de 1 US\$ por tonelada de $\mathrm{CO}_{2} \mathrm{e}$ en países como México, Ucrania y Polonia, hasta 127 US\$ por tonelada de $\mathrm{CO}_{2} \mathrm{e}$ en Suecia (World Bank, 2019). De manera similar, no existe un "precio de mercado" global para el carbono. El mercado está muy fragmentado geográficamente, y cada sistema de comercio de emisiones tiene sus propias 
características, como los gases cubiertos por el sistema, los sectores participantes, la asignación de los derechos de emisión, el permiso de usar créditos internacionales y la meta específica de reducción de GEI que el sistema debería ayudar a cumplir (ICAP, 2019).

En términos del volumen, el sistema de comercio de emisiones de la Unión Europea (EU ETS) es el más antiguo y el de mayores dimensiones a nivel internacional. En este sistema de comercio de derechos de emisión, el precio de cada crédito o tonelada de $\mathrm{CO}_{2} \mathrm{e}$ ha variado fuertemente desde un primer precio máximo de $30 €$ en el año 2005, una caída paulatina de precios hasta llegar a los 3,50 € en 2013, un estancamiento entre los 4 y los $8 €$ en los años posteriores y el fuerte incremento desde inicios del 2018 (Sandbag, 2020).

\subsection{Valoración monetaria según el coste de mitigación}

Según el enfoque del coste de mitigación, la valoración de las emisiones de GEI puede basarse en el coste de lograr un cierto nivel de reducción de esas emisiones mediante diferentes medidas (tecnológicas) de mitigación. La estimación del coste de mitigación de emisiones de GEI se refleja habitualmente en curvas de costes marginales de reducción (curvas MAC, por las siglas en inglés de Marginal Abatement Cost) que relacionan distintos grados de reducción en las emisiones con el coste marginal de dicha reducción. Las curvas MAC pueden construirse con base en la estimación de expertos o mediante su derivación de modelos (Kesicki, 2011). Las primeras implican el análisis de medidas de reducción individuales, mientras que las segundas son el resultado de modelos de la industria y del sector energético.

Las curvas MAC basadas en la opinión de expertos, más adecuadas a las circunstancias de las compañías que buscan reducir sus emisiones de GEI, analizan las tecnologías de reducción disponibles y el coste de cada una en un momento dado. Las tecnologías pueden abarcar desde medidas específicas de eficiencia energética, hasta plantar árboles o implementar procesos de captura y almacenamiento de carbono en plantas de generación eléctrica que usan combustibles fósiles. Cuando se colocan en orden ascendente de costes, estas tecnologías forman una curva MAC. Las curvas MAC están ligadas a un contexto temporal específico y descansan sobre una serie de supuestos, incluidos los relativos a la innovación tecnológica, la eficiencia de la implementación y el estado de la economía futura (Ekins et al., 2011).

Por ejemplo, en la curva estimada por McKinsey y Co. sobre el potencial de reducciones de emisiones de GEI a nivel global para el año 2030, cada una de las barras del histograma representa una tecnología diferente que podría adoptarse para reducir las emisiones (McKinsey y Co., 2009). El ancho de cada barra muestra el potencial de reducción de GEI de la tecnología en el año 2030, comparado con un valor fijado como base de referencia (business-as-usual), y la altura de cada barra muestra el coste por unidad de reducción de emisiones (1 tonelada de $\mathrm{CO}_{2} \mathrm{e}$ ), cuando la tecnología se instala al máximo potencial de mitigación. La curva de McKinsey y Co. se limita a las oportunidades técnicas de mitigación con un coste menor de $60 €$ por tonelada de $\mathrm{CO}_{2} \mathrm{e}$.

El enfoque del coste de mitigación es ampliamente utilizado en organizaciones del sector público y privado, incluidos los gobiernos y algunas grandes empresas de Europa (PwC, 2015). Cabe remarcar su utilidad para priorizar y diseñar posibles intervenciones dirigidas a reducir las emisiones de GEI, incluidas las opciones de evitar emisiones, pagar un impuesto sobre el carbono o comprar créditos de carbono. Sin embargo, el coste de mitigación refleja el coste financiero de reducir las emisiones en una cantidad determinada, y no el beneficio para la sociedad derivado de esa reducción de emisiones (ni el daño causado en caso de emitir la cantidad equivalente). El MAC específico varía para cada sector (y cada empresa dentro de ese sector). Las compañías que han hecho pocos esfuerzos para reducir las emisiones de GEI inicialmente pueden afrontar costes de reducción negativos (ahorros netos derivados de la 
implementación de opciones de reducción), mientras que las compañías que han agotado todas las opciones de reducción habituales pueden afrontar costes de reducción altos.

\subsection{Valoración monetaria según el coste social del carbono}

El enfoque del coste social del carbono (SCC, por las siglas en inglés de Social Cost of Carbon) valora las emisiones de GEI, teniendo en cuenta los impactos sobre el medio natural y los costes resultantes para la sociedad. En concreto, el SCC mide el valor monetario actual de emitir una unidad adicional de $\mathrm{CO}_{2}$ (o la cantidad equivalente de otros GEI), sumando todos los costes y beneficios cuantificables presentes y futuros.

El concepto del coste social del carbono es fundamental en la economía del cambio climático y es utilizado por los gobiernos de algunos países, como Estados Unidos y Canadá. Las estimaciones del coste social del carbono son complejas y generalmente utilizan modelos de análisis integrados (integrated assessment models) para evaluar los impactos biofísicos de las emisiones de GEI y valorar las posibles consecuencias en términos económicos. Estos modelos analizan simultáneamente factores científicos, sociales y económicos e integran básicamente cuatro elementos: la proyección de las futuras concentraciones atmosféricas de GEI, la proyección de futuros cambios climáticos, la proyección de los impactos ambientales y la valoración económica de los impactos ambientales (PwC, 2015). Los resultados de las más de 500 estimaciones del SCC existentes abarcan un rango de $-13,36$ a 2.386,91 US\$/t $\mathrm{CO}_{2} \mathrm{e}$, y varían debido a diferentes supuestos y valores para los parámetros usados en los diferentes modelos (Wang et al., 2019). Los altos grados de incertidumbre de las estimaciones del SCC están relacionadas con la comprensión científica del sistema climático, las suposiciones sobre las emisiones futuras y algunas cuestiones éticas e ideológicas, como la elección de la tasa de descuento, el grado de ponderación por equidad y el riesgo que la sociedad debería aceptar (Isacs et al., 2016). Cabe destacar especialmente las diferencias observadas en el uso de distintas tasas de descuento, uno de los aspectos más controvertidos de las estimaciones del SCC. Por ejemplo, mientras el Informe Stern defiende una tasa de descuento baja, con una tasa de preferencia en el tiempo cercana a cero, con el objetivo de no valorar menos el bienestar de las generaciones futuras que el de las generaciones presentes (Stern, 2007), Nordhaus (2016) considera diferentes tasas de descuento entre 2,5 y 5 por cien, basadas en el coste de oportunidad del capital.

La literatura académica más reciente afirma que muchas de las anteriores estimaciones del SCC son conservadoras y que las cifras resultantes subestiman los costes para el medio ambiente y la sociedad (High-Level Commission on Carbon Prices, 2017). Por ejemplo, Ackerman y Stanton (2012) exploran los efectos de ajustar la estimación para las principales incertidumbres que afectan los cálculos del SCC (la sensibilidad climática, el nivel de daños esperados con temperaturas bajas y altas, y la tasa de descuento), y obtienen valores entre $28 \mathrm{y}$ $96 \mathrm{US} \$ / \mathrm{t} \mathrm{CO} \mathrm{C}_{2} \mathrm{e}$ para una tasa de descuento de 3 por cien y entre 118 y $445 \mathrm{US} \$ / \mathrm{t} \mathrm{CO}_{2} \mathrm{e}$ para una tasa de descuento de 1,5 por cien (2007 US\$). Van den Bergh y Botzen (2014) reconocen la dificultad de calcular el "verdadero" valor del SCC debido a las limitaciones de los modelos de estimación existentes, y proponen un límite inferior de 125 US\$/t CO ${ }_{2}$ e (2014 US\$) basándose en un principio de precaución. Cabe remarcar que el amplio rango de incertidumbre de las estimaciones del SCC es similar a otras valoraciones de impacto, como por ejemplo, las valoraciones monetarias del daño causado por las emisiones de GEI a la salud humana, la calidad del ecosistema y los recursos sociales (Dong et al., 2019). 


\subsection{Comparación de los tres enfoques de valoración monetaria}

Las organizaciones pueden escoger entre tres enfoques posibles para asignar un valor monetario a los impactos causados por las emisiones de GEI, y la Tabla 1 resume las fortalezas y debilidades de cada alternativa. Mientras el precio de mercado y el coste de mitigación indican el coste privado de una empresa para comerciar con derechos de emisión o emprender un cambio tecnológico, el coste social del carbono expresa el valor monetario de los impactos causados por un incremento de emisiones de GEI. Por lo tanto, a pesar de la incertidumbre en torno a sus estimaciones, podemos decir que el enfoque del coste social del carbono es el que en mayor medida pretende recoger el valor destruido para el stakeholder medio ambiente (o su evitación) y, en consecuencia, para las generaciones presentes y futuras de la sociedad. En este sentido, el coste social del carbono parece el enfoque más compatible con el planteamiento de la contabilidad social del VSI.

\begin{tabular}{|c|c|c|c|}
\hline \multicolumn{4}{|c|}{$\begin{array}{l}\text { TABLA 1: CARACTERÍSTICAS DE LOS DIFERENTES ENFOQUES DE } \\
\text { VALORACIÓN MONETARIA }\end{array}$} \\
\hline & Precio de mercado & Coste de mitigación & Coste social del carbono \\
\hline Fortalezas & $\begin{array}{l}\text { - Es directamente } \\
\text { observable } \\
\text { - No se basa en } \\
\text { suposiciones sobre el } \\
\text { futuro }\end{array}$ & $\begin{array}{l}\text { - Se basa en costes conocidos } \\
\text { - Aunque se basa en } \\
\text { suposiciones sobre el futuro, } \\
\text { las estimaciones tienen } \\
\text { grados de incertidumbre } \\
\text { menores que el coste social } \\
\text { del carbono }\end{array}$ & $\begin{array}{l}\bullet \text { Expresa el valor } \\
\text { monetario de los } \\
\text { impactos (daños) } \\
\text { causados por un } \\
\text { incremento de } \\
\text { emisiones de GEI }\end{array}$ \\
\hline Debilidades & $\begin{array}{l}\text { - Representa una medida } \\
\text { de la cantidad a pagar } \\
\text { por las emisiones de } \\
\text { GEI en un escenario } \\
\text { político determinado, y } \\
\text { tiene poca relación con } \\
\text { los impactos de las } \\
\text { emisiones } \\
\text { - Varía } \\
\text { significativamente } \\
\text { entre países y regiones }\end{array}$ & $\begin{array}{l}\text { - Expresa el coste de reducir } \\
\text { una determinada cantidad de } \\
\text { emisiones de GEI, pero no } \\
\text { tiene relación con los } \\
\text { impactos evitados } \\
\text { - Se refiere a un objetivo de } \\
\text { reducción de emisiones } \\
\text { determinado } \\
\text { - Varía significativamente } \\
\text { entre sectores (y empresas) }\end{array}$ & $\begin{array}{l}\text { - Se basa en suposiciones } \\
\text { sobre el futuro, y las } \\
\text { estimaciones tienen } \\
\text { altos grados de } \\
\text { incertidumbre } \\
\text { - Los valores obtenidos } \\
\text { de las estimaciones } \\
\text { varían de manera } \\
\text { significativa, y } \\
\text { dependen fuertemente } \\
\text { de la tasa de descuento } \\
\text { empleada }\end{array}$ \\
\hline Valores & $\begin{array}{l}24,84 € / t \mathrm{CO}_{2} \mathrm{e}(\mathrm{EU} \text { ETS } \\
2019)\end{array}$ & $\begin{array}{l}5-49 € / t \mathrm{CO}_{2} \mathrm{e}^{*} \text { (precios de } \\
\text { carbono revelados por } \\
\text { empresas al CDP) }\end{array}$ & $\begin{array}{l}43-679 € / t \mathrm{CO}_{2} \mathrm{e}^{*} \\
\text { (estimaciones SCC de } \\
\text { Ackerman y Stanton, } \\
\text { 2012) }\end{array}$ \\
\hline $\begin{array}{l}\text { Ejemplos de } \\
\text { empresas }\end{array}$ & $\begin{array}{l}\text { Instalaciones afectadas } \\
\text { por el régimen } \\
\text { comunitario de comercio } \\
\text { de derechos de emisión } \\
\text { (EU ETS) }\end{array}$ & Microsoft, Royal DSM, SUEZ & $\begin{array}{l}\text { PUMA, Novo Nordisk, } \\
\text { Kering, Vodafone } \\
\text { Netherlands, } \\
\text { LafargeHolcim, BASF }\end{array}$ \\
\hline
\end{tabular}

Para ilustrar con ejemplos concretos la aplicación de los tres enfoques de valoración monetaria, revisamos a continuación algunos de los posibles valores de referencia. Como precio de mercado podemos considerar el precio de los derechos de emisión de $\mathrm{CO}_{2}$ en el mercado regulado europeo (EU ETS), que opera en 31 países (los 28 de la UE, más Islandia, 
Liechtenstein y Noruega) y limita las emisiones de más de 11.000 instalaciones de gran consumo de energía (centrales eléctricas y plantas industriales) y de las compañías aéreas que operan entre esos países (Comisión Europea, 2020). El precio medio anual en 2019 fue de 24,84 €/t CO 2 e (Sendeco2, 2020).

La obtención de un valor observable del coste de mitigación resulta más complicada. No obstante, en este caso podemos considerar los precios que asignan a las emisiones de $\mathrm{CO}_{2}$ las empresas que adoptan herramientas de internal carbon pricing con la finalidad de evaluar los riesgos y oportunidades relacionados con el clima (United Nations Global Compact, 2015). Estas empresas pueden utilizar diferentes enfoques para fijar un precio interno de carbono, entre ellos un análisis técnico de las medidas necesarias para alcanzar los objetivos de reducción de sus emisiones de GEI y los costes asociados. Al analizar el precio del carbono publicado por las grandes empresas que responden al cuestionario de Carbon Disclosure Project a nivel mundial, se puede apreciar que existe una gran dispersión en la distribución de precios entre países y sectores, con valores que oscilan entre entre 0,3 y 893 US\$/t $\mathrm{CO}_{2} \mathrm{e}$ (CDP, 2016). Sin embargo, alrededor del 80 por cien de los precios internos de carbono reportados varían entre 5 y 50 US\$/t CO CO $_{2}$ (4,93 a 49,29 € ajustado por inflación al año 20194). Ejemplos de empresas que están utilizando precios internos de carbono de estas magnitudes son Microsoft, Royal DSM y SUEZ. Cabe señalar que el reciente Informe de la Comisión de Alto Nivel sobre los Precios del Carbono estima que el nivel del precio de carbono requerido en 2020 para cumplir con los objetivos del Acuerdo de París ("mantener el aumento de la temperatura media mundial muy por debajo de $2{ }^{\circ} \mathrm{C}$ con respecto a los niveles preindustriales, y proseguir los esfuerzos para limitar ese aumento de la temperatura a $1,5^{\circ} \mathrm{C}^{\prime}$ ) es de $40-80 \mathrm{US} \$ / \mathrm{t} \mathrm{CO}_{2} \mathrm{e}$ (High-Level Commission on Carbon Prices, 2017).

Como posibles valores para el coste social del carbono podemos tomar el rango de estimaciones de Ackerman y Stanton (2012), actualizando los valores al año 2019. Si ajustamos estos valores por la inflación monetaria y la tasa de crecimiento del SCC a lo largo del tiempo (debido a que las emisiones de GEI perduran en la atmósfera y los daños causados aumentan con el tiempo), obtenemos valores entre 42,74 y 679,31 $€ / t \mathrm{CO}_{2} \mathrm{e} .{ }^{5}$ Las empresas que presentan una valoración monetaria de su impacto ambiental, ya sea en forma de EP\&L o de declaraciones integradas de impacto, suelen utilizar como proxy de monetización el coste social del carbono (PUMA, Novo Nordisk, Kering, Vodafone Netherlands, LafargeHolcim, BASF, etc.). A pesar de que la mayoría de empresas que revelan la fuente del valor monetario utilizado se basan en estimaciones del SCC con tasas de descuento bajas, los importes se sitúan por debajo del límite inferior de $122 € / t \mathrm{CO}_{2}$ e propuesto por Van den Bergh y Botzen (2014) (ajustado por inflación monetaria y la tasa de crecimiento).

La valoración monetaria del impacto de las emisiones de GEI - y presumiblemente la de otros impactos ambientales - se diferencia pues del proceso de monetización de las variables de valor social del VSI (Retolaza et al., 2016): mientras que la proxy más adecuado para aproximar el valor recibido por un stakeholder suele ser el precio de un producto o servicio sustitutivo en un mercado activo, en el caso de un impacto ambiental tenemos que recurrir a otras fuentes para reflejar la magnitud del impacto en las personas. Cabe remarcar que esta valoración monetaria no tiene que ver con el valor intrínseco de los elementos naturales, sino con el valor antropocéntrico de los servicios prestados por los ecosistemas a la sociedad.

\footnotetext{
${ }^{4}$ Conversión realizada sobre la base del tipo de cambio promedio anual de junio de 2015 a junio de 2016 indicado en CDP (2016) y ajustada por inflación mediante el índice de deflación del PIB mundial (Banco Mundial, 2020). ${ }^{5}$ Los datos han sido ajustados por inflación mediante el índice de deflación del PIB mundial (Banco Mundial, 2020) y un 3 por cien anual por crecimiento del daño causado en el tiempo (PwC, 2015).
} 


\section{CONCLUSIONES}

El presente trabajo contribuye a la literatura sobre contabilidad social y medioambiental y aporta una orientación práctica sobre cómo contabilizar el impacto ambiental de las organizaciones. La contabilidad social pretende ampliar la visión de la contabilidad financiera, tomando en consideración el valor que genera o destruye una organización más allá de su actividad económica y para todos sus stakeholders. El medio ambiente también puede ser considerado como un stakeholder de la organización, aunque no tenga conciencia para percibir el valor generado o destruido, ni voz para ser consultado al respecto. En su lugar, deberemos recurrir al conocimiento científico para determinar los impactos de la actividad organizativa sobre el entorno natural y las posteriores consecuencias para la sociedad en su conjunto.

La contabilización del valor ambiental es parecida a la contabilización del Valor Social Integrado (VSI), y engloba una primera fase de evaluación ambiental y una segunda fase de valoración del impacto. Sin embargo, una diferencia importante a tener en cuenta es la consideración de toda la cadena de valor, con el objetivo de reflejar todos los problemas ambientales relevantes causados de forma directa o indirecta por la empresa, aplicando el principio contable de materialidad. En cuanto a la asignación de un valor monetario a los impactos ambientales cuantificados, existen diferentes modelos disponibles, desde precios de mercado a costes de mitigación o costes sociales. Tras analizar las fortalezas y las debilidades de tres enfoques para asignar un valor a un particular problema ambiental, las emisiones de gases de efecto invernadero, concluimos que el coste social es la perspectiva que mejor se adecua al planteamiento del VSI. A diferencia de los enfoques del precio de mercado y del coste de mitigación, que indican los costes privados ocasionados para las empresas, el coste social expresa el valor monetario de los impactos causados sobre el medio ambiente y las personas. En conclusión, podemos afirmar que es posible integrar la valoración monetaria de los impactos ambientales en la contabilidad social, siempre y cuando se considera toda la cadena de valor y los costes (o beneficios) para la sociedad. La Figura 2 muestra esta integración de la valoración del impacto sobre el medio ambiente en el modelo poliédrico de análisis del VSI.

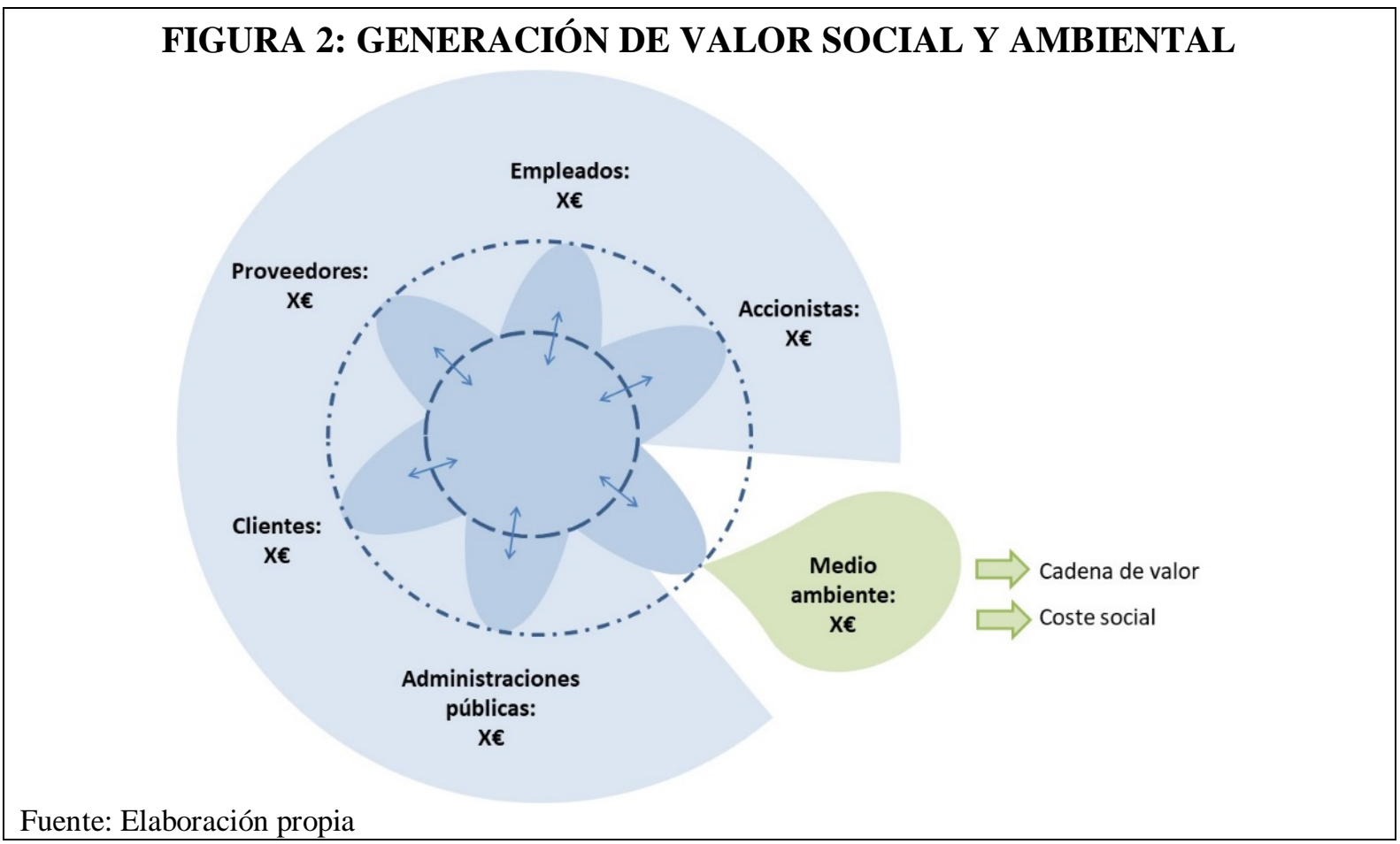


Por norma general, el valor ambiental generado por la actividad de una empresa afectará de forma negativa al medio ambiente y representará una "pérdida”. En el sistema económico actual, estas pérdidas no son costes reales que las empresas tengan que asumir, sino que se consideran externalidades. Sin embargo, es previsible que con el tiempo estos costes se internalicen gradualmente a través de diferentes instrumentos de política ambiental (tasas, impuestos, etc.). En este sentido, cuantificar el valor ambiental desde una perspectiva de contabilidad social tiene varias implicaciones prácticas para las empresas. El hecho de asignar un valor monetario concreto al impacto ambiental permite su integración con el impacto económico y social de la empresa y su transformación en indicadores útiles para la gestión interna y la comunicación externa. Por un lado, el proceso de valoración del impacto ambiental proporciona una manera de atender las demandas crecientes de mayor responsabilidad y transparencia y facilita el compromiso con las partes interesadas sobre cómo las empresas contribuyen a los Objetivos de Desarrollo Sostenible (ODS) de Naciones Unidas. La medición de la contribución a los ODS requiere que las organizaciones establezcan indicadores no solamente relacionados con sus operaciones, sino con sus impactos negativos y positivos, actuales y potenciales, sobre los diferentes ODS en toda su cadena de valor (GRI, et al., 2015). Por otro lado, cuantificar el valor ambiental de las empresas puede ayudar a transformar la contabilidad financiera convencional y mejorar la toma de decisiones interna de las empresas de acuerdo con principios de ética y sostenibilidad. El caso analizado de las emisiones de GEI muestra que incluso las empresas pioneras que adoptan herramientas de internal carbon pricing, suelen fijar precios por debajo del coste estimado para la sociedad. Los indicadores ambientales basados en los costes sociales, por lo tanto, pueden servir como un indicador del valor de los recursos naturales de los que dependen el éxito y la longevidad de la empresa. Finalmente, la información aportada por el valor ambiental no sólo sirve para reducir el impacto de las empresas sobre el medio ambiente. También permite a los responsables empresariales descubrir nuevas oportunidades para innovar en materia de diseño de productos, materiales y cadenas de suministro, y ayuda a identificar riesgos en los proveedores o en los mercados desde una perspectiva a largo plazo.

El presente trabajo se limita a analizar en profundidad la cuantificación y valoración monetaria de las emisiones de gases de efecto invernadero. Futuras líneas de investigación deberían analizar la valoración de otros impactos ambientales, como otras emisiones atmosféricas, consumo de agua, generación de aguas residuales, generación de residuos, etc., y evaluar la idoneidad de diferentes enfoques de valoración posibles para contabilizar el valor ambiental correspondiente. En particular, en aquellos casos en los que ya existe un precio establecido por el mercado o las administraciones públicas, se deberá estudiar en qué medida estos importes internalizan el impacto generado sobre el medio ambiente y la sociedad.

El trabajo ha argumentado que la valoración monetaria del impacto ambiental puede integrarse en la contabilidad social, y que puede contabilizarse como el valor generado o destruido para uno de los principales stakeholders de la organización, el medio ambiente. Sin embargo, no ha sido el objetivo de la investigación analizar cómo presentar esta información de manera compatible con la contabilidad financiera (Nicholls, 2020). En este sentido, la investigación futura debería explorar las sinergias y compatibilidades entre contabilidad financiera, social y ambiental y cómo estas contabilidades influyen en la estrategia de las empresas. A medida que se avance en la evaluación sistemática del impacto económico, social y ambiental de las empresas, será más evidente su papel en abordar los desafíos de sostenibilidad de la sociedad presente y futura. 


\section{BIBLIOGRAFÍA}

Ackerman, F. y Stanton, E.A. (2012): “Climate risks and carbon prices: Revising the social cost of carbon”, Economics, vol. 6, pp. 0-25.

Ayuso, S. (2018): La medición del impacto social en el ámbito empresarial, Cátedra MANGO de RSC, Documento de trabajo $n^{\circ} 26$, ESCI-UPF.

Banco Mundial (2020): https://datos.bancomundial.org/, consultado 13/03/2020.

Barbu, E. M., Dumontier, P., Feleaga, N. y Feleaga, L. (2014): "Mandatory environmental disclosures by companies complying with IAS/IFRS: The case of France, Germany, and the UK”, The International Journal of Accounting, vol. 49 n 2, pp. 231-247.

Bazin, D. y Ballet, J. (2004): "Corporate social responsibility: the natural environment as a stakeholder?”, International Journal of Sustainable Development, vol. 7 n 1 , pp. 5975.

Bebbington, J., Kirk, E. A. y Larrinaga, C. (2012): "The production of normativity: A comparison of reporting regimes in Spain and the UK", Accounting, Organizations and Society, vol. $37 \mathrm{n}^{0} 2$, pp. 78-94.

Bebbington, J. y Larrinaga, C. (2014): "Accounting and sustainable development: an exploration”, Accounting, Organizations and Society, vol. 39 n 6, pp. 395-413.

CDP (2016): Embedding a carbon price into business strategy, September 2016, Carbon Disclosure Project.

Comisión Europea (2020): https://ec.europa.eu/clima/policies/ets_es, consultado 17/06/2020.

Danish Environmental Protection Agency (2014): Assessment of potentials and limitations in valuation of externalities, Environmental Project No. 1561, Danish Ministry of the Environment.

Dong, Y., Hauschild, M., Sørup, H., Rousselet, R. y Fantke, P. (2019): "Evaluating the monetary values of greenhouse gases emissions in life cycle impact assessment", Journal of Cleaner Production, $\mathrm{n}^{0}$ 209, pp. 538-549.

Ekins, P., Kesicki, F. y Smith, A.P.Z. (2011): Marginal abatement cost curves: A call for caution, Energy Institute, University College London.

Elkington, J. (1997): Cannibals with Forks: The Triple Bottom Line of 21st Century Business, Oxford, Capstone.

Emerson, J., Bonini, S. y Brehm, K. (2003): The Blended Value Map: Tracking the Intersects and Opportunities of Economic, Social and Environmental Value Creation, William and Flora Hewlett Foundation, Menlo Park.

Faria, P. y Labutong, N. (2020): "A description of four science-based corporate GHG targetsetting methods”, Sustainability Accounting, Management and Policy Journal, vol. 11 $\mathrm{n}^{\mathrm{o}}$ 3, pp. 591-612.

Fernández, C., Fronti, L. y Scavone, G. (2018): Control económico de la gestión ambiental, Asociación Española de Contabilidad y Administración de Empresas (AECA), Madrid.

Freeman, R.E. (1984): Strategic management: A stakeholder approach, Pitman, Boston.

Freeman, R.E., Harrison, J.S., Wicks, A.C., Parmar, B.L. y de Colle, S. (2010): Stakeholder theory: The state of the art, Cambridge University Press, Cambridge.

Fullana, P. y Puig, R. (1997): Análisis del ciclo de vida, Rubes Editorial, Barcelona.

Gray, R., Owen, D. L. y Adams, C. A. (1996): Accounting and accountability: Changes and challenges in corporate social and environmental reporting, Prentice Hall, London.

Gray, R. (2002): "The social accounting project and Accounting Organizations and Society. Privileging engagement, imaginings, new accountings and pragmatism over critique?, Accounting, Organizations and Society, vol. 27 n 7 , pp. 687-708.

GRI, UN Global Compact y WBCSD (2015): SDG Compass: The guide for business action on the SDGs, GRI/UN Global Compact/WBCSD. 
Gröjer, J.E. y Stark, A. (1977): "Social accounting: a Swedish attempt", Accounting, Organizations and Society, vol. 2 n 4, pp. 349-386.

Haigh, N. y Griffiths, A. (2009): "The natural environment as a primary stakeholder: the case of climate change”, Business Strategy and the Environment, vol. 18 n $^{\circ}$ 6, pp. 347-359.

High-Level Commission on Carbon Prices (2017): Report of the High-Level Commission on Carbon Prices, World Bank, Washington, DC.

Hummel, K. (2019): "Reporting on the Sustainable Development Goals - Early evidence from Europe”, http://dx.doi.org/10.2139/ssrn.3411017

ICAP (2019): Emissions Trading Worldwide: Status Report 2019, International Carbon Action Partnership, Berlin.

IPCC (2007): Intergovernmental Panel on Climate Change Fourth Assessment Report.

IPCC (2014): AR5 Climate Change 2014: Impacts, Adaptation, and Vulnerability: Fifth Assessment Report of the Intergovernmental Panel on Climate Change, IPCC, Ginebra.

Isacs, L., Finnveden, G., Dahllöf, L., Håkansson, C., Petersson, L., Steen, B., Swanström, L. y Wikström, A. (2016): "Choosing a monetary value of greenhouse gases in assessment tools: A comprehensive review”, Journal of Cleaner Production, $n^{\circ} 127$, pp. 37-48.

Kering (2015): Environmental Profit \& Loss (EP\&L): Methodology and 2013 Group Results, Kering, París.

KPMG (2017): The KPMG Survey of Corporate Responsibility Reporting 2017. KPMG International Cooperative.

Kesicki, F. (2011): Marginal abatement cost curves for policy making - expert-based vs. modelderived curves, Updated in November 2011, Energy Institute, University College London.

Larrinaga, C., Moneva, J., Llena, F., Carrasco, F. y Correa, C. (2002): Regulación contable de la información medioambiental. Normativa española e internacional, Asociación Española de Contabilidad y Administración de Empresas (AECA), Madrid.

Larrinaga, C., Moneva, J.M. y Ortas, E. (2019): "Veinticinco años de contabilidad social y medioambiental en España: pasado, presente y futuro”, Revista Española de Financiación y Contabilidad, vol. 48 n $^{\circ}$ 4, pp. 387-405.

Lazkano, L. y Beraza, A. (2019): "Social accounting for sustainability: A study in the social economy”, Sustainability, vol. $11 \mathrm{n}^{0}$ 24, pp. 1-12.

Llena, F., Moneva, J. M. y Hernández, B. (2007): "Environmental disclosures and compulsory accounting standards: The case of Spanish annual reports”, Business Strategy and the Environment, vol. $16 \mathrm{n}^{\circ}$ 1, pp. 50-63.

Mandell, S. (2011): “Carbon emission values in cost benefit analyses”, Transport Policy, vol. $18 \mathrm{n}^{\circ} 6$, pp. 888-892.

McKinsey y Co. (2009): Pathways:to a low-carbon economy, Version 2 of the Global Greenhouse Gas Abatement Cost Curve.

Michelon, G., Pilonato, S. y Ricceri, F. (2015): "CSR reporting practices and the quality of disclosure: An empirical analysis”, Critical Perspectives on Accounting, vol. 33, pp. 5978.

Mitchell, R.K., Agle, B.R. y Wood, D.J. (1997): “Toward a theory of stakeholder identification and salience: defining the principle of who and what really counts", Academy of Management Review, vol. 22 n ${ }^{\circ}$ 4, pp. 853-886.

NCC (2016): Natural Capital Protocol Principles and Framework, Natural Capital Protocol.

Nicholls, J.A. (2020): "Integrating financial, social and environmental accounting", Sustainability Accounting, Management and Policy Journal, vol. $11 \mathrm{n}^{\circ}$ 4, pp. 745-769.

Nordhaus, W. (2016): "Revisiting the social cost of carbon”. PNAS, vol. 114 n $^{\circ}$ 7, pp. 15181523. 
Orts, E.W. y Strudler, A. (2002): “The ethical and environmental limits of stakeholder theory”, Business Ethics Quarterly, vol. 12 n² 2, pp. 215-233.

Parker, L.D. (2005): "Social and environmental accountability research: A view from the commentary box”, Accounting, Auditing \& Accountability Journal, vol. 18 n 6, pp. 842-860.

Phillips, R., Freeman, R.E. y Wicks, A.C. (2003): "What stakeholder theory is not”, Business Ethics Quarterly, vol. 13 no 4, pp. 479-502.

Phillips, R.A. y Reichart, J. (2000): "The environment as a stakeholder? A fairness-based approach”, Journal of Business Ethics, vol. 23 n 2, pp. 185-197.

Pistoni, A., Songini, L. y Bavagnoli, F. (2018): "Integrated reporting quality: An empirical analysis”, Corporate Social Responsibility and Environmental Management, vol. $25 \mathrm{n}^{\circ}$ 4, pp. 489-507.

Porter, M.E. y Kramer, M.R. (2011): “Creating shared value”, Harvard Business Review, vol. $89 \mathrm{n}^{\circ} 1 / 2$, pp. 62-77.

Pritchard, R. y van der Horst, D. (2018): Monetary Natural Capital Assessment in the Private Sector. A review of current status and research needs, Valuing Nature Natural Capital Synthesis Report VNP.

PUMA (2011): PUMA's Environmental Profit and Loss Account for the year ended 31 December 2010.

PwC (2015): Valuing corporate environmental impacts: Greenhouse gases, PwC methodology paper, Version 4.3., PricewaterhouseCoopers.

Retolaza, J.L. y San-Jose, L. (2011): "Social economy and stakeholder theory, an integrative framework for socialization of the capitalism”, CIRIEC-España, Revista de Economía Pública, Social y Cooperativa, no 73, pp. 193-213.

Retolaza, J.L. y San-Jose, L. (2018): “Contabilidad social para el bien común”, Revista de Responsabilidad Social de la Empresa, n 29, pp. 95-122.

Retolaza, J.L., San-Jose, L. y Ruíz-Roqueñi, M. (2016): Social accounting for sustainability: Monetizing the social value, Springer, Berlin, Heidelberg.

Richmond, B.J., Mook, L. y Jack, Q. (2003): “Social accounting for nonprofits: Two models”, Nonprofit Management and Leadership, vol .13 n 4, pp. 308-324.

Sandbag (2020): https://sandbag.be/index.php/carbon-price-viewer/, consultado 13/03/2020.

SBT (2019) https://sciencebasedtargets.org/, consultado 13/03/2020.

Sendeco2 (2020): https://www.sendeco2.com/es/precios-co2, consultado 13/03/2020.

Starik, M. (1995): "Should trees have managerial standing? Toward stakeholder status for nonhuman nature", Journal of Business Ethics, vol. 14 n 3, pp. 207-217.

Stern, N. (2007): The economics of climate change: The Stern Review, Cambridge University Press, Cambridge, UK.

Stone, C.D. (1974): Should trees have standing? Towards legal rights for natural objects, Kaufmann, Los Altos.

United Nations Global Compact (2015): Executive Guide to Carbon Pricing Leadership: A Caring for Climate Report, United Nations Global Compact.

Van den Bergh, J.C.J.M. y Botzen,W.J.W. (2014): “A lower bound to the social cost of $\mathrm{CO}_{2}$ emissions”, Nature Climate Change, vol. 4 n 4, pp. 253-258.

Van Kampen, M. (2018): Growing trend in Environmental Profit \& Loss Accounting: how to reap the benefits, Koninklijke Philips N.V.

Vodafone Netherlands (2015): Environmental Profit and Loss. Methodology and Results 2014/15.

Wang, P., Deng, X. Zhou, H. y Yu, Z. (2019): "Estimates of the social cost of carbon: A review based on meta-analysis”, Journal of Cleaner Production, vol. 209, pp. 1494-1507. 
Williams, A., Philipp, F., Kennedy, S. y Whiteman, G. (2017): “Systems thinking: a review of sustainability management research”, Journal of Cleaner Production, vol. 148, pp. 866881.

World Bank (2019) State and Trends of Carbon Pricing 2019, World Bank, Washington, DC.

WRI y WBCSD (2004): The Greenhouse Gas Protocol: A Corporate Accounting and Reporting Standard, World Resources Institute, Washington, D.C.

WWF (2018): Living Planet Report 2018: Aiming higher, WWF, Gland, Switzerland. 